\title{
Editorial: Computational Science and Engineering in 2020
}

\author{
Lorena A. Barba ${ }^{1}$ \\ ${ }^{1}$ Affiliation not available
}

October 7, 2020

In 1995, just one year after the founding of IEEE Computational Science $\&$ Engineering - the precursor of CiSE-founding editor-in-chief Ahmed H. Sameh wrote that the board's goal was "making this magazine the flagship of all aspects of computational science and engineering" (Sameh, 1995). What was it like at the time? 1995 marks the beginning of the dot-com boom. That year, Amazon and eBay first opened their digital doors, the Intel Pentium Pro was released, IBM unveiled Deep Blue, and the NumPy library for array computing in Python was first introduced. It was also the year that Peter Norvig and Stuart Russell published their classic textbook on Artificial Intelligence (Peter Norvig, 1995). And the University of Texas at Austin established its program in computational and applied mathematics, which J. Tinsley Oden announced in CSEEE (Oden, 1995). The dozen tenure-track positions opened with the new program and institute were housed in the departments of mathematics, computer science, engineering mechanics, aerospace engineering and other engineering specialties. It was conceived as a broadly interdisciplinary program, and that has been the hallmark of computational science and engineering across time.

The interdisciplinary essence of computational science has placed the field in the bewildering position of being central to vast swaths of new discoveries, while also lacking recognition and support to flourish. A PITAC report in 2005 declared that "much of the promise of computational science remains unrealized due to inefficiencies within the R\&D infrastructure and lack of strategic planning and execution" (Computational science: ensuring America's competitiveness, 2005). It called for supporting computational science as a "national imperative for research and education in the 21st century." The core hurdle was and is discipline-based research silos interfering with the integration of diverse skills and knowledge sets needed for advanced computational research. Quoting the report, "inadequate and outmoded educational structures within academia [...] leave computational science students to flounder amid competing departments." This was 10 years after the founding of UT Austin's new institute (now called the Oden Institute for Computational Engineering and Sciences, https://www.oden.utexas.edu), and 10 years before the Department of Computational Mathematics, Science and Engineering (CMSE) was established at Michigan State University (https://cmse.msu.edu). It was also the year when we faced up to the reality that the "free lunch" of continued increases in processor performance was over (Sutter, 2005). Clock speeds reached the $3 \mathrm{GHz}$ level - where they remain to this day - and major manufacturers turned to multicore architectures. The clock race ended and a mainstream 4-GHz processor never landed. "Concurrency really is hard," Herb Sutter wrote, and so it became harder to train computational scientists.

The landscape of computing soon became even more thorny, as programmable general-purpose GPUs hit the scene. In 2007, NVIDIA released the CUDA software development kit and the first graphics processors designed for computing. While the trend then called "GPGPU" picked up speed, the first highly influential applications of GPUs for deep learning were published, and a new era unleashed (Raina et al., 2009). Many of us went on long expeditions of adapting our best-loved algorithms for solving physical models formulated as differential equations to the new architecture and programming models. Some were decidedly successful, like those in the molecular modeling and quantum chemistry communities, for example. But for most it has either been a grind, or a trend to be avoided. In the last ten years, to cap all this, computational science 
was inundated with new approaches like machine learning and data-based models. Now, the trendy exploit is to try "physics-informed" neural networks in applications.

Computational science and engineering for many years was dominated by physical simulations based on mathematical modeling and numerical analysis. This is reflected in the articles CiSE published, on topics like computational electromagnetics, molecular dynamics for modeling macroscopic material properties, simulations of the earth's mantle convection-driven flow, quantum and molecular mechanics combined for chemistry applications, geographic environmental modeling (these, all in 1995). Scope broadened to areas like computation in medicine (2000, issue 5), data mining (2002, issue 4), and cloud computing (2009, issue 4). Notably, two special-issue themes were harbingers of topics trending today: Python for scientific computing (2007, issue 3), and reproducible research (2009, issue 1). In the first, some of CiSE's most highly cited articles appear, including the Matplotlib paper (Hunter, 2007) with more than 11,400 citations indexed by Google Scholar as of this writing. The theme reappeared in 2011 (issue 2), with an article on the NumPy array structure that has amassed more than 6,000 citations, so far. In more recent years, it has been hard to resist the onslaught of interest in machine learning and related topics. CiSE first covered machine learning in 2013 (issue 5), highlighting applications like materials sciences and climate informatics. The pull of computer science perspectives, versus computational science, began to sway the content before long. Do we need to reclaim focus?

As interim editor-in-chief for the year 2020, and now appointed EiC for the three-year period starting 2021, I have been reflecting on what focus I wish to bring to CiSE. Certainly, the early concerns about the hurdles of interdisciplinarity, and the educational needs of the new generations of computational scientists, are high on my list. Revisiting the role of the Python ecosystem in computational science seems timely now, with the recent convergence with GPU computing via projects to develop open-source libraries giving access to GPU devices from Python programs. In general, I aim for a heightened emphasis on open source research software, combined with attention for transparency and reproducibility. I was a member of the study committee of the National Academies that produced the consensus report on Replicability and Reproducibility in Science last year (NASEM, 2019), and it has been a long-time area of focus in my own research. Even if CiSE featured the topic in 2009 and 2012, it has trended mainstream only recently - it is time to integrate those considerations firmly into the publication process.

In 2017, Reproducible Research became a new peer-reviewed track for CiSE, with outgoing EiC George Thiruvathukal and myself as co-editors. There, we have spearheaded practices enhancing transparency and supporting open science, like asking reviewers if they want to participate in open peer review (open identity, and open report) and asking authors to post a preprint of their manuscript. Reviewers who opt-in have deposited their review reports in services like Figshare or Authorea (https://www.authorea.com/inst/18992), and authors cite the review reports with the preprint identifiers, acknowledging the reviewer contributions. This open-review process works within the existing systems, and was approved by the Computer Society VP of Publications in April 2018. I'm interested in expanding this model to other submissions, perhaps some special issues.

As to topical focus, my goal is to re-align CiSE with its origins of publishing "articles that help define the field of computational science and engineering, emphasizing significant computational contributions to science and engineering discipline"(citing first EIC Ahmed Sameh's launch editorial). A higher aspiration was alluded to by past EIC Francis Sullivan on occasion of the magazine's 20th anniversary, to be "a broad-based publication... about the fundamental role of computation in all aspects of human civilization" (Sullivan et al., 2018).

In this issue, CiSE tackles the historic menace to our way of life that is the COVID-19 pandemic, and the role of computing and data science in our battle against it. This is a milestone for CiSE, and it was made possible by the formidable efforts of editors, authors and reviewers to produce a high-quality issue with a breakneck timeline. The Computer Society recognizes this effort and the importance of the theme by making all these articles open access for six months. I hope they will be read widely and illuminate the power of computing as a scientific tool in the service of society. 
To close this editorial, I would like to recognize the new editors who accepted my invitation to join the board this year:

- Anne Elster (Novel Architectures), Professor of Computer Science and HPC, Norwegian University of Science and Technology

- Dirk Colby (Education), Director of HPC Studies, Computational Mathematics, Science and Engineering, Michigan State University

- Sharon Broude Geva (Education), Director of Advanced Research Computing, University of Michigan

- Michaela Taufer, Dongarra Professor in High Performance Computing, University of Tennessee Knoxville

- Karla Morris (Software Engineering), Principal Member of Technical Staff at Sandia National Laboratories

- Ilkay Altintas (Data Track), Chief Data Science Officer at the San Diego Supercomputer Center, UCSD

- Anna-Karin Tornberg, Professor of Numerical Analysis, Sweedish Royal Institute of Technology

- Ewa Deelman, Principal Scientist, USC Information Sciences Institute, University of Southern California

And starting January 2021, we welcome:

- Kelly Gaither (Visualization Corner), Director of Visualization at the Texas Advanced Computing Center (TACC), University of Texas

- Karen Willcox, Professor of Aerospace Engineering and Engineering Mechanics and Director of the Oden Institute for Computational Engineering and Sciences, University of Texas

- John M. Shalf (Leadership Computing), Department Head for Computer Science, Lawrence Berkeley National Laboratory

- Kathryn Mohror (Leadership Computing), Computer Scientist at Lawrence Livermore National Laboratory

I look forward to working with all of them, and our continuing editors, to realize the aspirations for CiSE to be the flagship of all aspects of computational science and engineering.

\section{References}

Looking Back, Looking Forward: Evaluation and Vision. (1995). IEEE Computational Science 83 Engineering, 2, 1. https://doi.org/10.1109/MCSE.1995.10010

Artificial Intelligence: A Modern Approach.. (1995). Prentice Hall.

UT Austin establishes Computational and Applied Mathematics program. (1995). IEEE Computational Science and Engineering, 2(2), 7-9. https://doi.org/10.1109/99.388942

(2005). President's Information Technology Advisory Committee, Arlington, VA. https://apps.dtic.mil/ sti/pdfs/ADA462840.pdf

The free lunch is over: A fundamental turn toward concurrency in software. (2005). Dr. Dobb's Journal, $30(3), 202-210$.

Large-scale deep unsupervised learning using graphics processors. (2009). Proceedings of the 26th Annual International Conference on Machine Learning - ICML 09, 873-880. https://doi.org/10.1145/1553374. 1553486 
Matplotlib: A 2D Graphics Environment. (2007). Computing in Science ES Engineering, 9(3), 90-95. https://doi.org/10.1109/mcse.2007.55

Reproducibility and Replicability in Science. (2019). National Academies Press. https://doi.org/10. $17226 / 25303$

Former CiSE EICs Reflect on the Magazines 20th Anniversary. (2018). Computing in Science E Engineering, 20(1), 3-7. https://doi.org/10.1109/mcse.2018.011111118 